\title{
Calculation of Rotor Dynamic Coefficients for Labyrinth Seals*
}

\author{
B.P. WILLIAMS ${ }^{a}$ and R.D. FLACK ${ }^{b, \dagger}$ \\ ${ }^{a}$ Baker and Botts, Dallas, TX 75201-2980, USA; ${ }^{\mathrm{b}}$ Mechanical, Aerospace, and Nuclear Engineering, \\ Rotating Machinery and Controls Laboratory, University of Virginia, Charlottesville, VA 22903-2441, USA
}

(Received 13 November 1997; In final form 29 January 1998)

\begin{abstract}
A single control volume, Iwatsubo based bulk flow method for the calculation of these coefficients is developed and implemented. The method herein uses a unique iterative technique to first identify the mass flow rate based on pressure drops across the individual teeth, which is then used in the governing sets of continuity and momentum equations. The method is applicable to different teeth geometries and arrangements. A parametric analysis of the effect of mass flow rate on rotor dynamic coefficients is performed and suggests that a small variation in mass flow rate does not significantly detract from the accuracy of the predicted dynamic coefficients; the mass flow rate calculation implemented in this paper is sufficiently accurate. Furthermore, the inclusion of some tangential momentum parameters has been previously proposed to improve the accuracy of the Iwatsubo method. However, from the current analysis the inclusion of such parameters is also shown to have little effect on the rotor dynamic coefficients and does not lead to improved correlation with experimental data. Comparisons to experimental data suggest that the method herein is reasonable for use as a design tool to predict the trends and actual values of cross-coupled stiffness, the most important seal parameter in rotor dynamic analyses. The method is also shown to be useful in predicting the order of magnitude of principal stiffness and damping coefficients.
\end{abstract}

Keywords: Seals, Labyrinth seals, Rotor dynamics

\section{INTRODUCTION}

Labyrinth seals, which are typically used in centrifugal compressors, accelerate and decelerate a compressible fluid through a tortuous path to dissipate energy and reduce the amount of leakage flow. Common types of labyrinth seals include straight-through, staggered, stepped, interference, and combined seals. A straight-through labyrinth seal is shown in Fig. 1.

Nonuniform circumferential pressure distributions within the labyrinth chambers can exert forces on the rotating shaft. As with hydrodynamic journal bearings as reviewed by Flack and

* This paper was originally presented at ISROMAC-7.

${ }^{\dagger}$ Corresponding author. Tel.: 804 924-7421. Fax: 804 982-2037. E-mail: rdf@virginia.edu. 


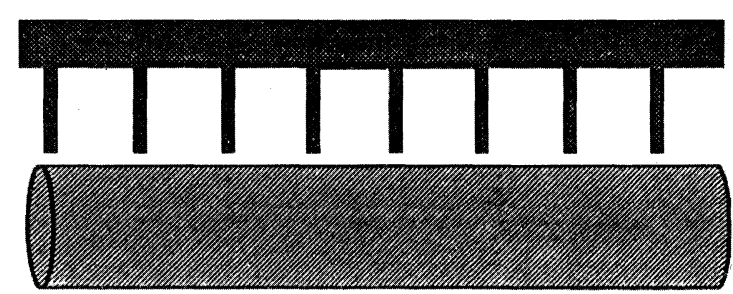

FIGURE 1 Straight-through labyrinth seal.

Kostrzewsky (1993), such forces can cause a rotor to become unstable. In an effort to include the fluid induced forces in rotordynamic analyses, many researchers have sought rotordynamic coefficients for labyrinth seals.

The accurate prediction of these rotordynamic coefficients for labyrinth seals is the subject of this paper. Straight-through teeth-on-stator and teethon-rotor seals as well as interlocking seals will be investigated. Because the cross-coupled stiffness is the more significant parameter used in rotordynamic analyses, particular attention is paid to this potentially destabilizing stiffness coefficient.

While accurate calculation of the leakage flow is important in the prediction of rotordynamic coefficients, primary importance will be placed here on prior investigations concerning dynamic coefficients rather than leakage flow. The idea that a labyrinth seal could exert a destabilizing force on a rotor was first promulgated by Alford (1965), who used a simple one-dimensional model which neglected the circumferential flow in the labyrinths. Destabilizing forces were predicted only if the labyrinths converge.

The current work can be classified into three categories: (1) single control volume analyses; (2) multi-control volume analyses; and (3) computational fluid dynamic analyses. Single control volume analyses, while varying somewhat from each other, all have the underlying simplification that the flow is separated into two parts: a leakage flow (jet flow), and a circumferential flow (core flow). The control volume is the labyrinth chamber which includes the area of intersection between the leakage flow and the circumferential flow as shown in Fig. 2.

Jenny (1980) used a 3-D computational fluid dynamic (CFD) model to construct a simple single

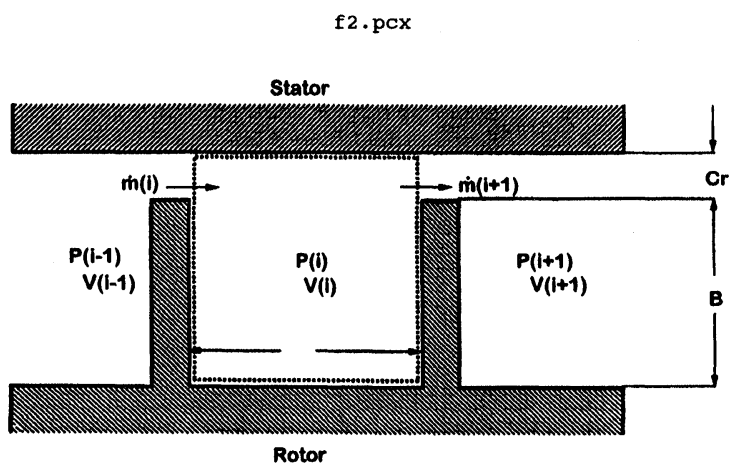

FIGURE 2 2-D view cavity control volume.

control volume model with the three-dimensional flow in the circumferential direction replaced with a single core flow of mean circumferential velocity. The most important feature of this analysis is the introduction of a tangential momentum coefficient which describes the amount of circumferential momentum imparted to a chamber from the leakage flow.

Iwatsubo (1980) included the time dependency of area change due to a change in rotor position. The resulting equations were linearized and solved by a finite difference approach and, later by Iwatsubo et al. (1982), by an analytical separation of variables approach from which stiffness and damping coefficients were calculated. Childs and Scharrer (1986a) extended Iwatsubo's analysis by including the variation of area in the circumferential direction due to eccentricity. This analysis employs a different solution format for the resulting equations utilizing a "reduced circumferential momentum" equation. Kanki and Morii (1986) employed Iwatsubo's method but included the tangential momentum coefficient introduced by Jenny (1980) and also introduced a dynamic-state tangential momentum parameter of his own.

Wyssmann et al. (1984) introduced the first two control volume approach. Wyssmann et al. accounted for the shear stresses between the core flow and the jet flow by using two control volumes for the circumferential flow. One control volume was used for the bulk of the labyrinth chamber and one was used in the area of the leakage flow. 
Scharrer (1988) extended the theory of Childs and Scharrer (1986a) by using the same two control volumes as Wyssmann but including the recirculation velocity in the analysis. He reports better correlation with experimental data than the single control volume analysis of Childs and Scharrer (1986a). Nordmann and Weiser (1990) used a three control volume technique similar to the two control volume method. In this case the control volume within the jet flow was subdivided into two regions: the region above the labyrinth chamber and the region above the labyrinth fin.

Most of the computational fluid dynamic analysis of labyrinth seals has been in conjunction with bulk flow models. Jenny used a 3-D finite difference model to help in the development of his bulk flow model. Scharrer (1988) used results of the simulation of compressible flow in a single labyrinth cavity to develop his recirculation model. Nordmann and Weiser (1988) used a 3-D finite difference method to calculate the dynamic coefficients of seals for a centered shaft.

The first extensive experimental investigation of the destabilizing force caused by a labyrinth seal was performed by Benckert and Wachter (1980). The seals were tested for two distinct cases: no seal rotation but with fluid prerotation, and seal rotation but with no fluid prerotation. The static stiffness coefficients and cross-coupled force were presented. These experiments showed that the circumferential velocity is the origin of the crosscoupled stiffness and, therefore, an important parameter in modeling.

Childs and Scharrer (1986b) performed tests of teeth-on-rotor and teeth-on-stator seals for 16 teeth seals. This is the first testing in which direct damping coefficients were obtained. The rotor speed and pressure drops are consistent with those used in industrial machinery. Childs and Scharrer (1988) extended their earlier experiments and performed tests of the same seals but for speeds up to $16000 \mathrm{rpm}$. The increased speed is important since it shows a speed dependency of the crosscoupled stiffness which was not apparent at lower speeds. The published data for these experiments are well-documented.
For this effort a single control volume analysis was developed to calculate the leakage properties and stiffness and damping coefficients of straightthrough labyrinths for both teeth-on-rotor and teeth-on-stator configurations as well as interlocking configurations. One purpose of this paper is to compare the dynamic coefficients calculated by the method to the data published by Childs and Scharrer (1988) in order to assess the accuracy of the model. Second, for this paper several techniques are applied to alleviate stability problems on iterative methods and matrix inversion illconditions often encountered for these geometries. Third, in an effort to more accurately model the flow characteristics within the seal and obtain better predictions of the dynamic coefficients, a tangential momentum parameter is implemented. Fourth, a unique and improved method of calculating the flow rate is presented and parametric studies on the effect of mass flow calculations on dynamic coefficients are performed to determine the reasonableness of using existing techniques for approximating mass flow rate.

\section{THEORY}

The solution technique consists in deriving the continuity and momentum equations for each labyrinth cavity representing a single control volume similar to that performed by Childs and Scharrer (1986a). A leakage model is used to account for the axial leakage from one chamber to the next. The resulting set of partial differential equations is linearized using a perturbation analysis for small motion about a centered position. The zeroth order equations are solved for the pressure and velocity in each cavity. The temporal and spatial derivatives in the first order equations are eliminated by assuming an elliptical shaft orbit and resulting responses for the pressure and velocity oscillations. The resulting set of linear algebraic equations is solved for the pressure and velocity perturbations about the circumference. The dynamic coefficients are determined by integrating the pressure perturbations around and 
along the shaft. Details of the method can be found in Williams (1992).

The three dimensional flow in a labyrinth seal is very complex. In order to create a set of tractable equations which describe the flow, simplifying assumptions must be made: (1) The circumferential velocity and pressure within a labyrinth chamber are constant in the axial direction and are functions of angular position only in the perturbed case. (2) The temperature within each seal cavity is constant. (3) The gas is assumed to be ideal. (4) Pressure variations within a cavity are negligible relative to the pressure difference across a seal tooth. (5) The eccentricity of the rotor is small relative to the radial seal clearance. (6) The acoustic resonance frequency of a cavity is much higher than that of the rotational frequency. (7) The axial component of velocity is negligible for the determination of the circumferential shear stress. (8) The shear stress contribution to the stiffness and damping coefficients is negligible.

A set of equations can be developed which approximately describes the flow within a labyrinth seal. Principles of conservation of mass and conservation of circumferential momentum are applied to each labyrinth chamber which serves as a control volume as shown in Figs. 2 and 3 for a teeth-on-rotor labyrinth. The resulting continuity equation is as follows:

$$
\frac{\partial}{\partial t}\left(\rho_{i} A_{i}\right)+\frac{1}{R_{\mathrm{s}}} \frac{\partial}{\partial \theta}\left(\rho_{i} V_{i} A_{i}\right)+\dot{m}_{i+1}-\dot{m}_{i}=0,
$$

where $A_{i}$ is the transverse surface area and $\dot{m}_{i}$ and $\dot{m}_{i+1}$ are axial mass flow rates per circumferential length entering and exiting the chamber. The resulting circumferential momentum equation is:

$$
\begin{gathered}
\frac{\partial\left(\rho_{i} V_{i} A_{i}\right)}{\partial t}+\frac{1}{R_{\mathrm{s}}} \frac{\partial\left(\rho V_{i}^{2} A_{i}\right)}{\partial \theta}+\dot{m}_{i+1} V_{i}-\dot{m}_{i} V_{i-1} \\
=-\frac{A}{R_{\mathrm{s}}} \frac{\partial P}{\partial \theta}+\tau_{\mathrm{r} i} \operatorname{ar}_{i} L_{i}-\tau_{\mathrm{s} i} \operatorname{as}_{i} L_{i}
\end{gathered}
$$

where, for example, for teeth-on-stator, $\operatorname{ar}_{i}=1$, $\mathrm{as}_{i}=\left(2 B_{i}+L_{i}\right) / L_{i}$. Childs and Scharrer (1986a)

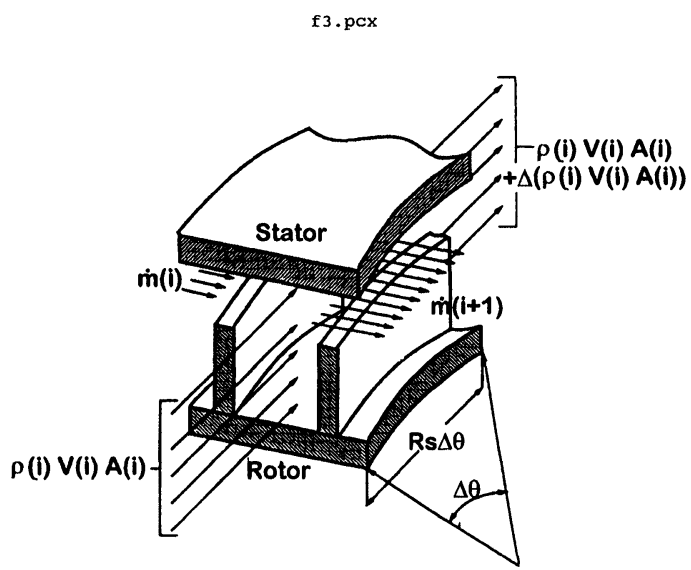

FIGURE 3 3-D view cavity control volume.

modeled the shear stress in the labyrinth chamber as turbulent flow in a smooth pipe. The typical resulting equation for rotor shear stresses is

$$
\begin{aligned}
\tau_{\mathrm{r} i}= & \frac{\rho_{i}}{2}\left(R \omega-V_{i}\right)^{2} n_{0}\left(\frac{\left|R_{\mathrm{s}} \omega-V_{i}\right| \mathrm{Dh}}{\nu}\right)^{m_{0}} \\
& \times \operatorname{sgn}\left(R_{\mathrm{s}} \omega-V_{i}\right),
\end{aligned}
$$

where $\mathrm{Dh}_{i}$ is the hydraulic diameter. The constants $m_{0}$ and $n_{0}$ are given for turbulent flow between smooth annular surfaces as $m_{0}=-0.25$ and $n_{0}=0.079$.

In order to solve these equations, a perturbation analysis is performed with the eccentricity ratio $\varepsilon=e / C_{\mathrm{r}}$ serving as the perturbation parameter. Perturbation variables consist of a static component (zeroth order) denoted by the superscript 0 and a varying component (first order) denoted by the superscript 1. For example, $P_{i}=P_{i}^{0}+\varepsilon P_{i}^{1}$ and $V_{i}=V_{i}^{0}+\varepsilon V_{i}^{1}$.

These variables are used with the above equations and like orders of the perturbation parameter $\varepsilon\left(\varepsilon^{0}, \varepsilon^{1}\right)$ are grouped. The zeroth order equation $\left(\varepsilon^{0}\right)$ determines the circumferential velocity and pressure distribution for a centered position along the length of the seal. In the centered case, pressure and velocity are constant within a given chamber and vary only from chamber to chamber. The zeroth order equation also defines the mass 
flow leakage rate. The first order equation $\left(\varepsilon^{1}\right)$ determines the pressure and velocity perturbations resulting from the eccentricity of the rotor. In the eccentric case, the velocity and pressure perturbations are not constant within a given chamber but are functions of circumferential position $(\theta)$ and time. These pressure fluctuations are responsible for the forces applied to the rotor by the seal.

The zeroth order leakage rate is therefore determined as discussed below and the zeroth order pressure distribution is found in conjunction with this leakage calculation. The zeroth order circumferential momentum equation is:

$$
\dot{m}^{0}\left(V_{i}^{0}-V_{i-1}^{0}\right)=\left(\tau_{\mathrm{ri}}^{0} \mathrm{ar}_{i}-\tau_{\mathrm{si}}^{0} \mathrm{as}_{i}\right) L_{i} .
$$

With the mass flow rate determined, the only unknowns in this equation are the shear stresses and circumferential velocity. Since the shear stresses are functions of circumferential velocity and known variables only, the above equation can be solved by an iterative technique for the circumferential velocity in each chamber if the circumferential velocity at the entrance of the seal is specified.

The first order continuity and momentum equations resulting from grouping coefficients of $\varepsilon^{1}$ are:

$$
\begin{aligned}
& G_{1 i} \frac{\partial P_{i}^{1}}{\partial t}+G_{2 i} \frac{\partial P_{i}^{1}}{\partial \theta}+G_{3 i} \frac{\partial V_{i}^{1}}{\partial t}+G_{4 i} \frac{\partial V_{1}^{i}}{\partial \theta}+G_{5 i} P_{i}^{1} \\
& +G_{6 i} P_{i-1}^{1}+G_{7 i} P_{i+1}^{1}=G_{8 i} H_{i}^{1}+G_{9 i} \frac{\partial H_{i}^{1}}{\partial t}+G_{10 i} \frac{\partial H_{i}^{1}}{\partial \theta}
\end{aligned}
$$

$$
\begin{gathered}
X_{1 i} \frac{\partial V_{i}^{1}}{\partial t}+X_{2 i} \frac{\partial V_{i}^{1}}{\partial \theta}+X_{3 i} \frac{\partial P_{i}^{1}}{\partial \theta}+X_{4 i} V_{i}^{1}+X_{5 i} V_{i-1}^{1} \\
+X_{6 i} P_{i}^{1}+X_{7 i} P_{i-1}^{1}+X_{8 i} P_{i+1}^{1}=X_{9 i} H_{i}^{1}
\end{gathered}
$$

The coefficients $G_{i}$ and $X_{i}$ in the above equations are either constants or zeroth order variables. Perturbation equations for these seals often lead to near singular matrices. To avoid such singularities the perturbation equations were formulated based on an expression for mass flow rate which did not require the use of axial velocity as a variable as shown by Williams (1992); the matrices resulting from this formulation were always well conditioned. The spatial and temporal derivatives are eliminated by assuming a synchronous elliptical orbit for the rotor and similar pressure and velocity fluctuations. Substitution and grouping like terms of sines and cosines leads to a set of eight linear simultaneous equations for each cavity. The equations can be solved for pressure and velocity perturbations caused by the eccentricity of the rotor. Once the pressure fluctuations are known, the rotordynamic coefficients of the following form can be calculated:

$$
-\left\{\begin{array}{c}
F_{x} \\
F_{y}
\end{array}\right\}=\left[\begin{array}{cc}
K & k \\
-k & K
\end{array}\right]\left\{\begin{array}{c}
X \\
Y
\end{array}\right\}+\left[\begin{array}{cc}
C & c \\
-c & C
\end{array}\right]\left\{\begin{array}{c}
\dot{X} \\
\dot{Y}
\end{array}\right\} .
$$

For the assumed elliptical orbit, the rotor displacements and velocities can be written and one can also find the dynamic forces of similar form. The $X$ and $Y$ force components can be found byintegrating the pressure around the seal and the dynamic coefficients can be determined from the forces. Using the perturbation analysis leads to the grouping of like terms and yields the final solutions to the stiffness and damping coefficients.

\section{IMPLEMENTATION OF METHOD}

In order to implement the theory, the mass flow rate through the seal must be determined for the zeroth order. The amount of leakage is of importance for reasons other than those concerning dynamic coefficients and a considerable amount of work has been published on leakage flow calculation.

The leakage through a labyrinth seal is typically modeled as an adiabatic throttling process. A pressure drop takes place in each of the annular spaces as a result of an assumed isentropic expansion. The velocity resulting from this expansion is completely lost in the following chamber for 
an ideal labyrinth. For straight-through labyrinths typically used in industry, a portion of the kinetic energy associated with each stage is carried over into the next stage.

The method treats one restriction at a time and does not give an overall equation for leakage flow. This local approach accounts for different geometries for each restriction and includes the velocity carry-over from one restriction to the next. The leakage can be determined through an iterative process by linking together the pressure losses due to each restriction to determine a total pressure loss. This approach also allows for a more realistic assessment of choked flow. Details of the method can be found in Williams (1992) and is more accurate than that used by others in that a closed form equation with restricting assumptions is not used to calculate the mass flow rate of the entire seal.

According to Benvenuti et al. (1980), the mass flow rate crossing the $i$ th throttling is given by

$$
\begin{aligned}
M_{i}= & K_{\mathrm{f} i} A_{\mathrm{g}} \rho_{i-1} r_{i}^{1 / \gamma} \\
& \times \sqrt{\alpha W_{i-1}^{2}+\frac{2 \gamma R T_{i-1}}{\gamma-1}\left(1-r_{i}^{(\gamma-1) / \gamma}\right)}
\end{aligned}
$$

for subsonic speeds, and the speed of sound is reached in the $i_{\text {th }}$ throttling chamber when

$$
r_{i} \leq\left[\frac{2}{\gamma+1}+\frac{\alpha(\gamma-1) W_{i-1}^{2}}{2 \gamma R T_{i-1}}\right]^{\gamma /(\gamma-1)},
$$

where the axial velocity is defined as

$$
W_{i-1}=\frac{M_{i} R T_{i-2}}{K_{\mathrm{f} i-1} P_{i-2} r^{1 / \gamma} A} .
$$

Based on the calculated mass flow rate, the pressure ratio across each throttling can be found. The procedure, as depicted graphically in Fig. 4, is as follows.

First an initial guess for mass flow rate based on Vermes (1961) method is obtained. Then using this mass flow rate, the pressure drop is calculated for each restriction and a seal exit pressure is determined. If this pressure matches (within some reasonable tolerance) the specified exit pressure then the mass flow rate and pressure distribution are determined. If the exit pressure does not match, then the mass flow rate is adjusted accordingly and the process is repeated until the correct exit pressure is obtained.

In addition to the zeroth order mass flow rate and pressure distribution, the coefficients $G_{i}$ and $X_{i}$ in the first order continuity and momentum equations are needed to determine the rotordynamic coefficients. Once these $G_{i}$ 's and $X_{i}$ 's are determined, a system of equations may be developed and solved. The solution of this system of equations leads directly to the rotordynamic coefficients. The equations are in matrix form and formulated by substitution of the perturbation variables and grouping like orders of $\varepsilon$.

In the perturbation of mass flow rate, the expression given by Neumann (1964) is employed:

$$
\dot{m}_{i}=\mu_{1 i} \mu_{2} H_{i} \sqrt{\frac{\left(P_{i-1}^{2}-P_{i}^{2}\right)}{R T}},
$$

where Neumann assumed an isothermal case. While the isothermal assumption may not be completely accurate, this expression allows the perturbation of mass flow rate without introducing the axial velocity as an additional perturbation variable. This expression is employed by Iwatsubo (1980) and Childs and Scharrer (1986a), in their single control volume analyses. In this case it is used only for the perturbation of mass flow rate and not the actual calculation of mass flow. In the continuity equation $\dot{m}_{i+1}-\dot{m}_{i}$ is determined by using the following simplification suggested by Childs and Scharrer (1986a):

$$
\dot{m}_{i+1}-\dot{m}_{i}=\frac{\dot{m}_{i+1}^{2}-\dot{m}_{i}^{2}}{2 \dot{m}^{0}}
$$

Using the above relation:

$$
\begin{aligned}
& \dot{m}_{i+1}-\dot{m}_{i}=\frac{\mu_{2}^{2} H^{2}}{2 \dot{m}^{0} R T} \\
& \quad \times\left[\mu_{1 i+1}^{2}\left(P_{i}^{2}-P_{i+1}^{2}\right)-\mu_{1 i}^{2}\left(P_{i+1}^{2}-P_{i}^{2}\right)\right]
\end{aligned}
$$


f 4 . pcx



FIGURE 4 Iteration method for finding mass flow rate.

and substituting $P=P_{i}^{0}+\varepsilon P_{i}^{1}$ leads to the determination of the unknown coefficients.

\section{EFFECT OF MASS FLOW CALCULATION ON DYNAMIC COEFFICIENTS}

A parametric study of the effect of mass flow rate on dynamic coefficients was performed. The dynamic coefficients are determined based on the calculated mass flow rate (nominal) and mass flow rates of $50 \%, 90 \%, 110 \%$, and $200 \%$ of the nominal value for various operating conditions. The results give an indication of the sensitivity of dynamic coefficients to mass flow rate. Because of its importance in rotordynamic analysis, particular attention is paid to cross-coupled stiffness. Conditions for these cases are presented in Table I and replicate some of the parameters of Childs and Scharrer (1988).
TABLE I Operating conditions for example case

\begin{tabular}{lc}
\hline Operating Conditions & $300000 \mathrm{~N} / \mathrm{m}^{2}$ \\
Inlet Pressure & $94300 \mathrm{~N} / \mathrm{m}^{2}$ \\
Exit Pressure & $283.15^{\circ} \mathrm{K}$ \\
Inlet Temperature & \\
Fluid Properties & Air \\
Type of Gas & 1.40 \\
Specific Heat Ratio & $287 \mathrm{~J} / \mathrm{kg}-{ }^{\circ} \mathrm{K}$ \\
Gas Constant & \\
Seal Geometry & 16 \\
Number of Teeth & $0.0756 \mathrm{~m}$ \\
Radius of Shaft & $0.00033 \mathrm{~m}$ \\
Zeroth Order Clearance & $0.003175 \mathrm{~m}$ \\
Pitch of Seal Strips & $0.003175 \mathrm{~m}$ \\
Height of Seal Strip & $0.0001524 \mathrm{~m}$ \\
Tooth-Tip Width & STATOR \\
Location of Teeth &
\end{tabular}

Typical results of a parametric study for crosscoupled stiffness are shown in Fig. 5. This and other figures show that calculations based on mass flow rates of $50 \%$ and $200 \%$ of the nominal mass 


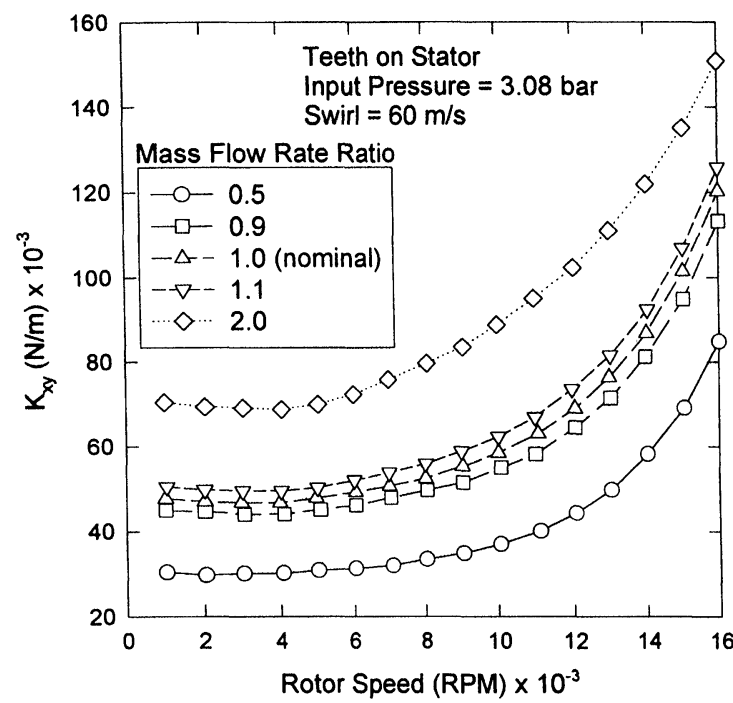

FIGURE 5 Effect of mass flow rate on cross coupled stiffness.

flow rate lead to cross-coupled stiffness values which are significantly different from the values obtained with the nominal mass flow rate. For high preswirl and teeth-on-rotor configurations, the differences become less pronounced with increasing speed while for the other operating conditions the differences increase with speed. The figures also show that, while a large variance in mass flow rate $(50 \%$ and $200 \%)$ from the nominal values leads to a large variance in crosscoupled stiffness, a small difference in mass flow rate does not significantly alter the calculated values for cross-coupled stiffness. The results of this analysis indicates that small differences $(10 \%$ or less) between actual and calculated mass flow rates should not detract significantly from the accuracy of the cross-coupled stiffness calculation.

A similar parametric analysis was performed for principal stiffness for the above case and is presented in Fig. 6. The figure shows that principal stiffness is fairly insensitive to mass flow rate. The sensitivity increases with rotor speed with differences in principal stiffness of approximately $40 \%$ at the highest speed for large variances from the nominal mass flow rate $(50 \%$ and $200 \%$ cases). The analysis indicates that small differences

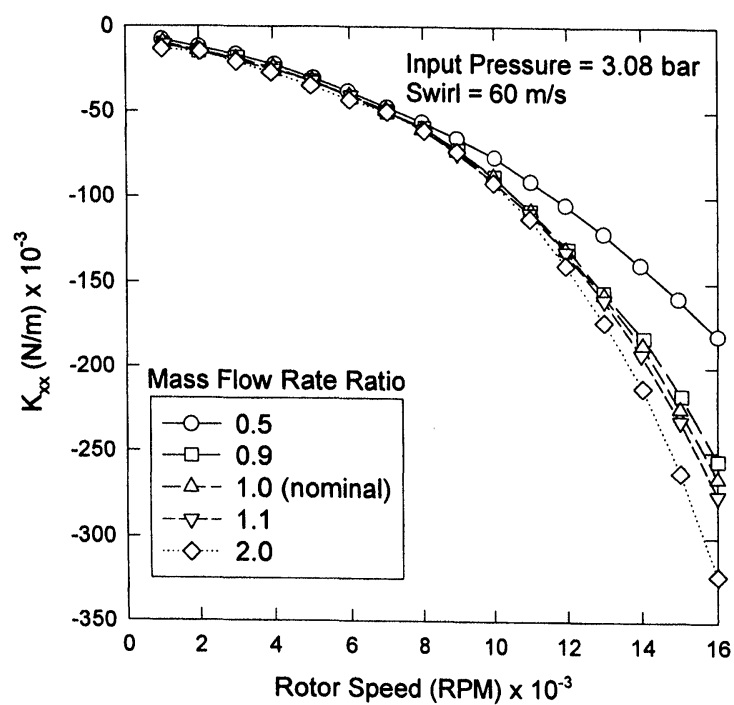

FIGURE 6 Effect of mass flow rate on principal stiffness.

between calculated and actual mass flow rates should not significantly affect the principal stiffness calculation while more significant differences in the mass flow rate could somewhat affect the stiffness calculation.

\section{COMPARISON TO EXPERIMENTAL DATA}

In order to assess the applicability of using the present analysis as a design tool, predicted values for rotordynamic coefficients are compared to experimental data.

A typical comparison to the data of Benckert and Wachter (1980) and predictions of Childs and Scharrer (1986a) is given in Fig. 7. Dimensionless cross-coupled stiffness is plotted against dimensionless input circumferential velocity (preswirl) and definitions of dimensionless parameters are given in Benckert and Wachter (1980). The calculated values show a good correlation with the experimental data and better agreement than the predictions from Childs and Scharrer (1986a). A linear increase in dimensionless cross-coupled stiffness with increase in dimensionless preswirl is shown. 


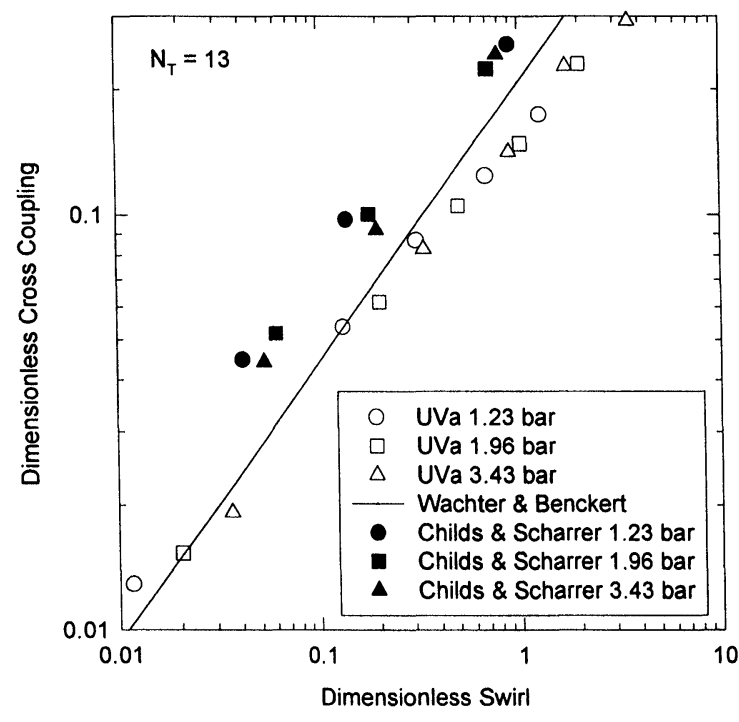

FIGURE 7 Comparison of predicted cross coupling stiffness to Benckert and Wachter (1980) and Childs and Scharrer (1986a).

A typical comparison to experimental data of calculated cross-coupled stiffness versus circumferential velocity ratio is given in Fig. 8. Comparisons are made for low and high rotor speeds. The calculated values show that cross-coupled stiffness increases linearly with circumferential velocity ratio for both low and high rotational speeds. These trends were seen for both teeth-on-rotor and teeth-on-stator labyrinths. The experimental data of Childs and Scharrer (1988) corroborate these trends with good correlation in most cases studied.

Since a small variation in circumferential velocity ratio leads to a large variation in cross-coupled stiffness, accurate measurement of this parameter is important for the above comparisons. The circumferential velocity was calculated as described in Childs and Scharrer (1988) based on the angle of the inlet guide vanes and the mass flow rate. Unfortunately, no error estimates are given for the measurement of this parameter. Slight differences in actual and measured circumferential velocities could cause large differences in crosscoupled stiffness; therefore, differences between

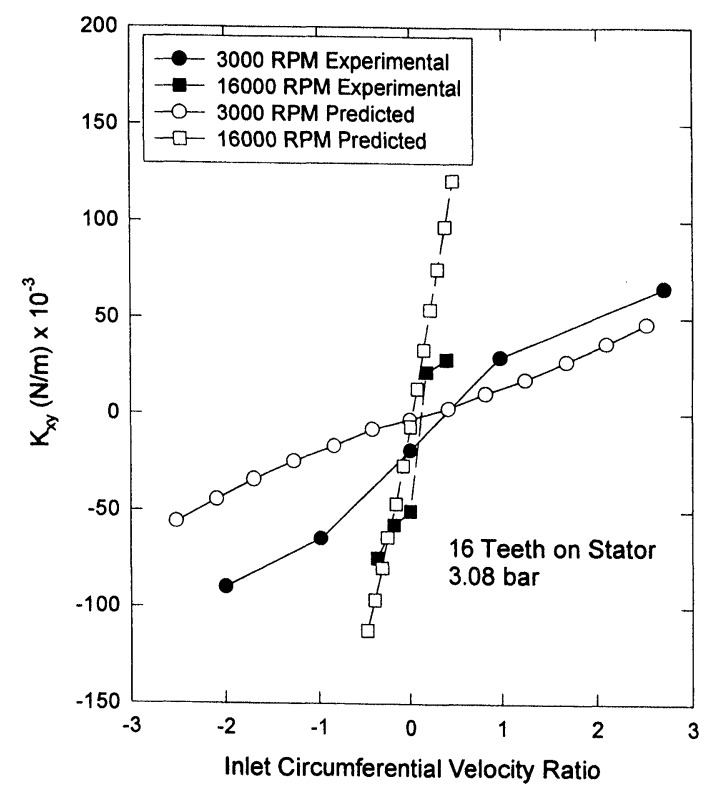

FIGURE 8 Comparison of predicted cross coupled stiffness to data of Childs and Scharrer (1988).

calculated and measured values can be partially attributed to uncertainty in the test conditions in addition to the approximations inherent in the method.

A typical comparison to experimental data of calculated cross-coupled stiffness versus rotor speed is given in Fig. 9 for teeth on the stator. Experimental results show slightly decreasing stiffness with increasing speed. Theoretical results show increasing stiffness with increasing speed. The trends of experimental data and calculated values are dissimilar. Furthermore, as with the cross-coupled stiffness versus circumferential velocity plot, the actual values of cross-coupled stiffness differ. The discrepancies can be attributed to the reasons discussed above.

Typical principal stiffness comparisons are made with the data of Childs and Scharrer (1988). A comparison to experimental data of calculated principal stiffness versus rotor speed is given in Fig. 10. The calculations show a sharp decrease of stiffness with increasing speed while the experimental data show fairly constant values. This 


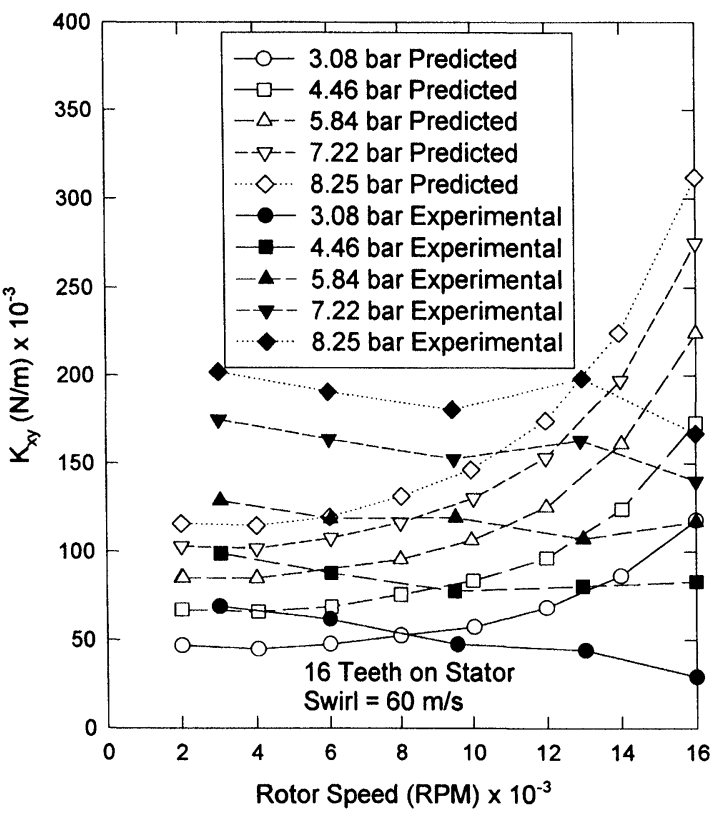

FIGURE 9 Comparison of predicted cross coupled stiffness to data of Childs and Scharrer (1988) same trend of sharp decrease in stiffness with speed is also predicted by Childs and Scharrer's analytical method. In addition to poor correlation with trends, the actual calculated values of principal stiffness generally differ significantly from the experimental values.

A typical comparison to Childs and Scharrer's experimental data of calculated principal damping versus inlet circumferential velocity ratio is given in Fig. 11. For low speeds, calculated values are positive with a slight increase in damping with increasing preswirl for positive preswirls and a slight increase in damping with decreasing preswirl for negative preswirls. For high speeds, calculated values show an increase in damping with increasing circumferential velocity ratio. Measured values show the same trend as calculated for low speeds. For high speeds, the experimental data do not show as consistent trends with respect to circumferential velocity ratio. As with the principal stiffness, a

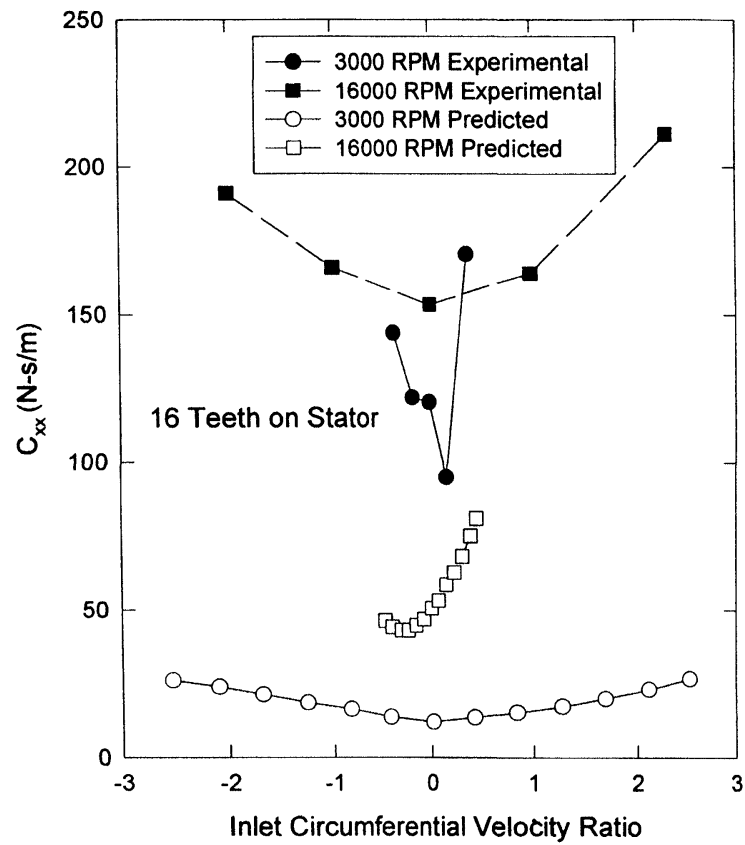

FIGURE 11 Comparison of predicted principal damping to data of Childs and Scharrer (1988)
FIGURE 10 Comparison of predicted principal stiffness to

data of Childs and Scharrer (1988).

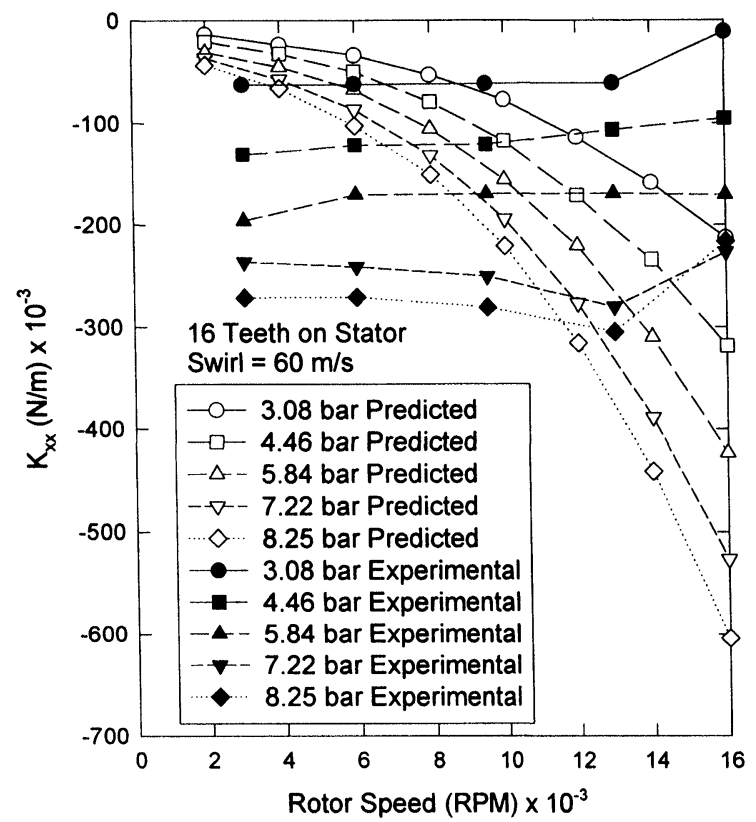


large offset exists between the actual predicted and experimental values.

\section{INCORPORATION OF TANGENTIAL MOMENTUM PARAMETER}

Through a CFD investigation, Jenny (1980) developed the following relationship to describe the momentum imparted by the leakage flow to the tangential flow on passage through the labyrinth chamber:

$$
m k_{\mathrm{j}}\left(\operatorname{Vin}_{i}^{0}-V_{i}^{0}\right)=\dot{m}\left(\operatorname{Vin}_{i}^{0}-\operatorname{Vout}_{i}^{0}\right)
$$

Jenny's tangential momentum parameter, $k_{\mathrm{j}}$, was determined to be approximately 0.15 for teeth-onstator seals, 0.35 for teeth-on-rotor seals, and 0.90 for interlocking seals. Almost complete mixing occurs with interlocking seals and the mean circumferential velocity is approximately equal to the exit circumferential velocity. This expression may be used without modification in the zeroth order circumferential momentum equation. For the first order circumferential momentum equation the terms $\left(V_{i}^{1}-V_{i-1}^{1}\right)$ and $\left(V_{i}^{0}-V_{i-1}^{0}\right)$ occur and are used in the approximation of the circumferential momentum crossing the boundaries of the labyrinth chamber. The above theory is implemented by replacing $\left(V_{i}-V_{i-1}\right)$ in the circumferential momentum equation with the appropriate form of Jenny's tangential momentum parameter. These modifications lead to a revised form for the perturbation coefficients.

The effect of incorporating these tangential momentum parameters is illustrated by comparing the dynamic coefficients calculated with and without the tangential momentum parameters to experimental data with an emphasis on crosscoupled stiffness. Cross-coupled stiffness predicted with and without incorporation of the tangential momentum parameters is plotted along with experimental data. Fig. 12 shows typical crosscoupled stiffness versus inlet circumferential velocity ratio. The use of the tangential parameters (denoted by "+ TMP" on the plot) results in the

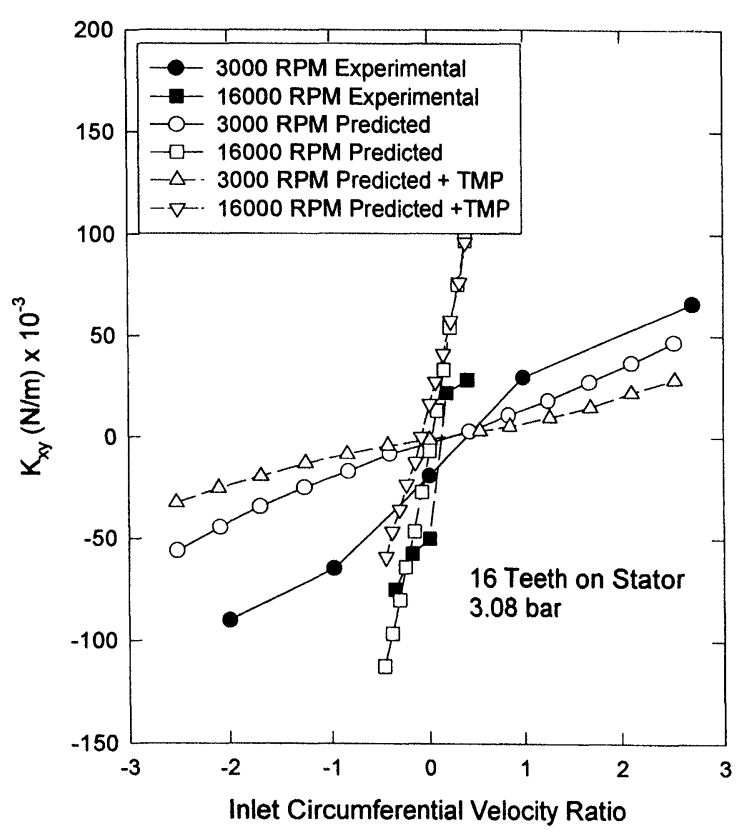

FIGURE 12 Effect of tangential momentum parameter on cross coupled stiffness.

same trends as without their use and does not lead to better correlation with experimental data. For low speeds, the use of the tangential parameters actually makes the comparison to experimental data worse. Based on these representative results, it does not appear that the use of the tangential momentum parameters, as implemented improves the prediction of cross-coupled stiffness.

\section{CONCLUSIONS}

A modified Iwatsubo method for the calculation of rotordynamic coefficients for labyrinth gas seals was implemented. The mass flow rate calculation makes use of a relation between mass flow rate through a single annular orifice and the pressure drop across the orifice. An iteration on mass flow rate is performed until the sum of pressure drops due to all orifices matches the specified pressure drop across the seal. Perturbation equations for these seals often lead to near singular matrices, 
which lead to unreasonable values for the rotordynamic coefficients. To avoid this problem the perturbation equations were formulated based on an expression for mass flow rate which did not require the use of axial velocity as a variable. The matrices resulting from this formulation were well conditioned.

A parametric analysis on the effect of mass flow rate calculation on dynamic coefficients was performed. The results of this analysis show that, for the cases tested, a small variation between the calculated and experimental mass flow rates should not significantly detract from the accuracy of the dynamic coefficient calculation and that the revised mass flow rate calculation is sufficiently accurate.

Comparisons to experimental data of crosscoupled stiffness, principal stiffness, and principal damping were performed to assess the reasonableness of using this analysis as a design tool. The results of these comparisons show that, for the most part, the trends of cross-coupled stiffness with most of the operating conditions tested compare well with experimental data. The actual values of cross-coupled stiffness were shown to be accurate within about a factor of two. Better correlation occurred in many cases. The comparisons to data suggest that the method is reasonable for use as a design tool to predict the trends and actual values of cross-coupled stiffness, the most important seal parameter in rotordynamic analyses. Trends and actual values for principal stiffness and principal damping did not compare as well with experimental data as did the crosscoupled stiffness. Principal stiffness and damping terms are accurate only to about an order of magnitude although correlation is better in many cases.

In an attempt to achieve better correlation with experimental data, tangential momentum parameters were incorporated into the present analysis. Unfortunately the use of these parameters showed little effect on the dynamic coefficients and in some cases made the comparisons to data worse.

\section{NOMENCLATURE}

$\begin{array}{ll}A & \text { transverse area } \\ \text { ar } & \quad \text { dimensionless length upon which shear } \\ & \text { acts on rotor } \\ \text { as } & \quad \text { dimensionless length upon which shear } \\ & \quad \text { acts on stator } \\ B & \text { height of cavity } \\ C & \text { principal damping } \\ c & \text { cross coupled damping } \\ C_{\mathrm{r}} & \text { nominal radial seal clearance } \\ \text { Dh } & \text { hydraulic diameter } \\ e & \text { eccentricity } \\ F & \text { force } \\ G_{i} & \text { continuity perturbation coefficients } \\ H & \text { local radial clearance } \\ K, K_{x x} & \text { principal stiffness } \\ k, K_{x y} & \text { cross coupled stiffness } \\ K_{\mathrm{f}} & \text { orifice flow coefficient } \\ k_{\mathrm{j}} & \text { Jenny's tangential momentum parameter } \\ L & \text { length of cavity } \\ \dot{m} & \text { leakage mass flow rate } \\ M & \text { mass flow rate (Benevenuti) } \\ m_{0}, n_{0} & \text { empirical parameters for shear } \\ P & \text { pressure } \\ r & \text { pressure ratio } \\ R_{\mathrm{S}} & \text { shaft radius } \\ R & \text { ideal gas constant } \\ T & \text { temperature } \\ V & \text { velocity } \\ W & \text { axial component of velocity } \\ X & x \text { position } \\ X_{i} & \text { momentum perturbation coefficients } \\ Y & y \text { position } \\ \alpha & \text { empirical constant } \\ \varepsilon & \text { perturbation parameter, } e / C_{\mathrm{r}} \\ \gamma & \text { specific heat ratio } \\ \rho & \text { density } \\ \theta & \text { circumferential position } \\ \tau & \text { shear } \\ \mu_{1}, \mu_{2} & \text { empirical constants for mass flow } \\ & \text { calculation } \\ & \text { kinematic viscosity } \\ & \text { rotor rotational speed } \\ & \end{array}$




\author{
Subscripts \\ $i \quad i$ th chamber
}

\section{Superscripts}

$0,1 \quad$ order of component

\section{Acknowledgments}

The research was supported by the Rotating Machinery and Controls Laboratory (ROMAC) at the University of Virginia. The authors also thank Dr. Mitchel Rosen for his helpful discussions.

\section{References}

Alford, J.S. (1965) Protecting turbomachinery from self-excited rotor whirl, Trans. ASME J. of Engineering for Power, 87, 333-344.

Benckert, H. and Wachter, J. (1980) Flow induced spring coefficients of labyrinth seals for application in rotor dynamics, NASA Conf. Pub. 2133: Rotordynamic Instability Problems in High-Performance Turbomachinery, Texas A\&M Workshop, Texas, pp. 189-212.

Benvenuti, E., Ruggeri, G. and Tomasini, E.P. (1980) Analytical and experimental development of labyrinth seals for process centrifugal compressors, Performance Prediction of Centrifugal Pumps and Compressors, 25th ASME Gas Turbine Conference, New Orleans, pp. 21-34.

Childs, D.W. and Scharrer, J.K. (1986a) An Iwatsubo-based solution for labyrinth seals: comparison to experimental results, Trans. ASME J. of Engr. for Gas Turbines and Power, 108, 325-331.

Childs, D.W. and Scharrer, J.K. (1986b) Experimental rotordynamic coefficient results for teeth-on-rotor and teeth-onstator labyrinth gas seals, Trans. ASME J. of Engineering for Gas Turbines and Power, 108, 599-604.

Childs, D.W. and Scharrer, J.K. (1988) Theory versus experiment for the rotordynamic coefficient of labyrinth gas seals: Part II - A comparison to experiment, Trans. ASME J. of
Vibration, Acoustics, Stress, and Reliability in Design, 110 281-287.

Flack, R.D., Kostrzewsky, G.J. and Taylor, D.V. (1993) A hydrodynamic journal bearing test rig with dynamic measurement capabilities, Tribology Transactions, 36, 497-512

Iwatsubo, T. (1980) Evaluation of instability forces of labyrinth seals in turbines or compressors, NASA Conf. Pub. 2133 . Rotordynamic Instability Problems in High-Performance Turbomachinery, Texas A\&M Workshop, Texas, pp. 139-168.

Iwatsubo, T. Motooka, N. and Kawai, R. (1982) Flow induced force of labyrinth seal, NASA Conf. Pub. 2250: Rotordynamic Instability Problems in High-Performance Turbomachinery, Texas A\&M Workshop, Texas, pp. 205-222.

Jenny, R. (1980) Labyrinths as a cause of self-excited rotor oscillations in centrifugal compressors, Sulzer Technical Review, 4, 149-156.

Kanki, H. and Morii, S. (1986) Destabilizing force of labyrinth seal, NASA Conf. Pub. 2443: Rotordynamic Instability Problems in High-Performance Turbomachinery, Texas A\&M Workshop, Texas, pp. 205-224.

Neumann, K. (1964) Zur Frage der Verwendung von Durchblickdichtungen im Dampfturbinenbau, Maschinenbautechnik, 13, pp. 188-195.

Nordmann, R. and Weiser, P. (1988) Rotordynamic coefficients for labyrinth seals calculated by means of a finite difference technique, NASA Conf. Pub. 3026: Rotordynamic Instability Problems in High-Performance Turbomachinery, Texas A\&M Workshop, Texas, pp. 161-176.

Nordmann, R. and Weiser, P. (1990) Evaluation of rotordynamic coefficients of look-through labyrinths by means of three volume bulk flow model, NASA Conf. Pub. 3122 . Rotordynamic Instability Problems in High-Performance Turbomachinery, Texas A\&M Workshop, Texas, pp 147-164.

Scharrer, J.K. (1988) Theory versus experiment for the rotordynamic coefficients of labyrinth gas seals: Part I - A two control volume model, Trans. ASME J. of Vibration, Acoustics, Stress, and Reliability in Design, 110, 270-280.

Vermes, G. (1961) A fluid mechanics approach to the labyrinth seal leakage problem, Trans. of the ASME J. of Engineering for Power, 83, 161-169.

Williams, B.P. (1992) The calculation of rotordynamic coefficients for labyrinth seals, M.S. Thesis, University of Virginia

Wyssmann, H.R., Pham, T.C. and Jenny, R.J. (1984) Prediction of stiffness and damping coefficients for centrifugal compressor labyrinth seals, ASME Trans. J. Engineering for Gas Turbines and Power, 106, 920-926. 


\section{ait \\ ENERGY MATERIALS}

M A N E Y publishing

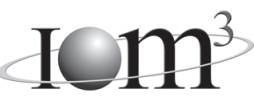

\section{Materials Science \& Engineering for Energy Systems}

Maney Publishing on behalf of the Institute of Materials, Minerals and Mining

The Institute of Materials, Minerals \& Mining

Economic and environmental factors are creating ever greater pressures for the efficient generation, transmission and use of energy. Materials developments are crucial to progress in all these areas: to innovation in design; to extending lifetime and maintenance intervals; and to successful operation in more demanding environments. Drawing together the broad community with interests in these areas, Energy Materials addresses materials needs in future energy generation, transmission, utilisation, conservation and storage. The journal covers thermal generation and gas turbines; renewable power (wind, wave, tidal, hydro, solar and geothermal); fuel cells (low and high temperature); materials issues relevant to biomass and biotechnology; nuclear power generation (fission and fusion); hydrogen generation and storage in the context of the 'hydrogen economy'; and the transmission and storage of the energy produced.

As well as publishing high-quality peer-reviewed research, Energy Materials promotes discussion of issues common to all sectors, through commissioned reviews and commentaries. The journal includes coverage of energy economics and policy, and broader social issues, since the political and legislative context influence research and investment decisions.

\section{CALL FOR PAPERS}

Contributions to the journal should be submitted online at http://ema.edmgr.com

To view the Notes for Contributors please visit: www.maney.co.uk/journals/notes/ema

Upon publication in 2006, this journal will be available via the Ingenta Connect journals service. To view free sample content online visit: www.ingentaconnect.com/content/maney

For further information please contact:

Maney Publishing UK

Tel: +44 (0)113 2497481 Fax: +44 (0)1132486983 Email: subscriptions@maney.co.uk

or

Maney Publishing North America

Tel (toll free): 8662975154 Fax: 6173546875 Email: maney@maneyusa.com

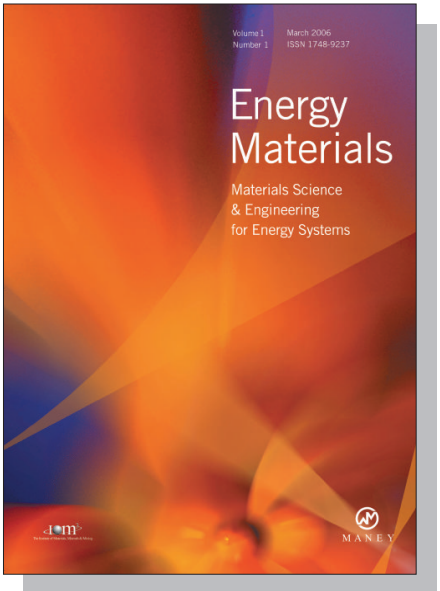

EDITORS

Dr Fujio Abe

NIMS, Japan

Dr John Hald, IPL-MPT, Technical University of Denmark, Denmark

Dr R Viswanathan, EPRI, USA

\section{SUBSCRIPTION INFORMATION}

Volume 1 (2006), 4 issues per year

Print ISSN: 1748-9237 Online ISSN: 1748-9245

Individual rate: $£ 76.00 / U S \$ 141.00$

Institutional rate: $£ 235.00 /$ US $\$ 435.00$

Online-only institutional rate: $£ 199.00 / U S \$ 367.00$

For special $\mathrm{IOM}^{3}$ member rates please email

subscriptions@maney.co.uk

\section{For further information or to subscribe online please visit www.maney.co.uk}



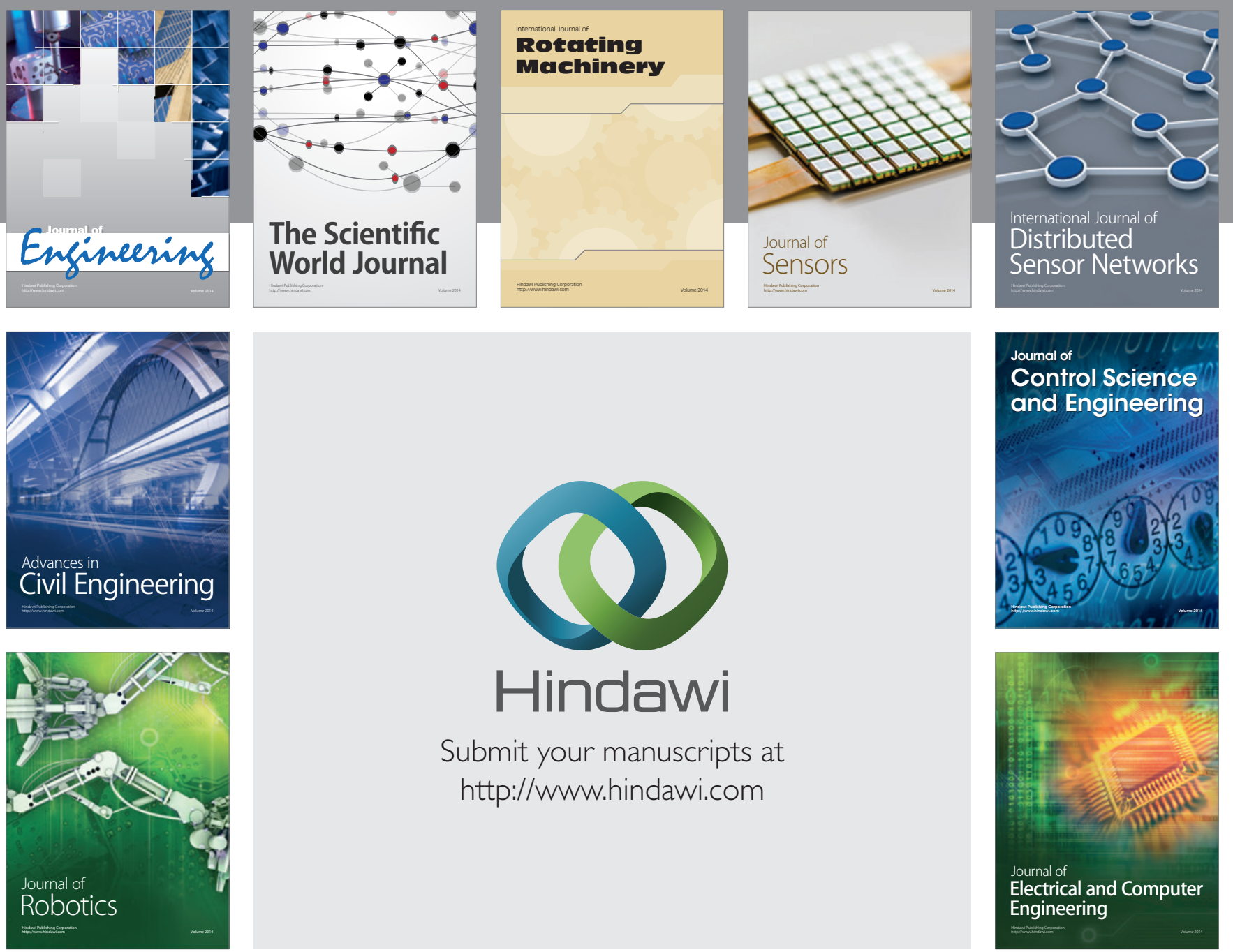

Submit your manuscripts at

http://www.hindawi.com
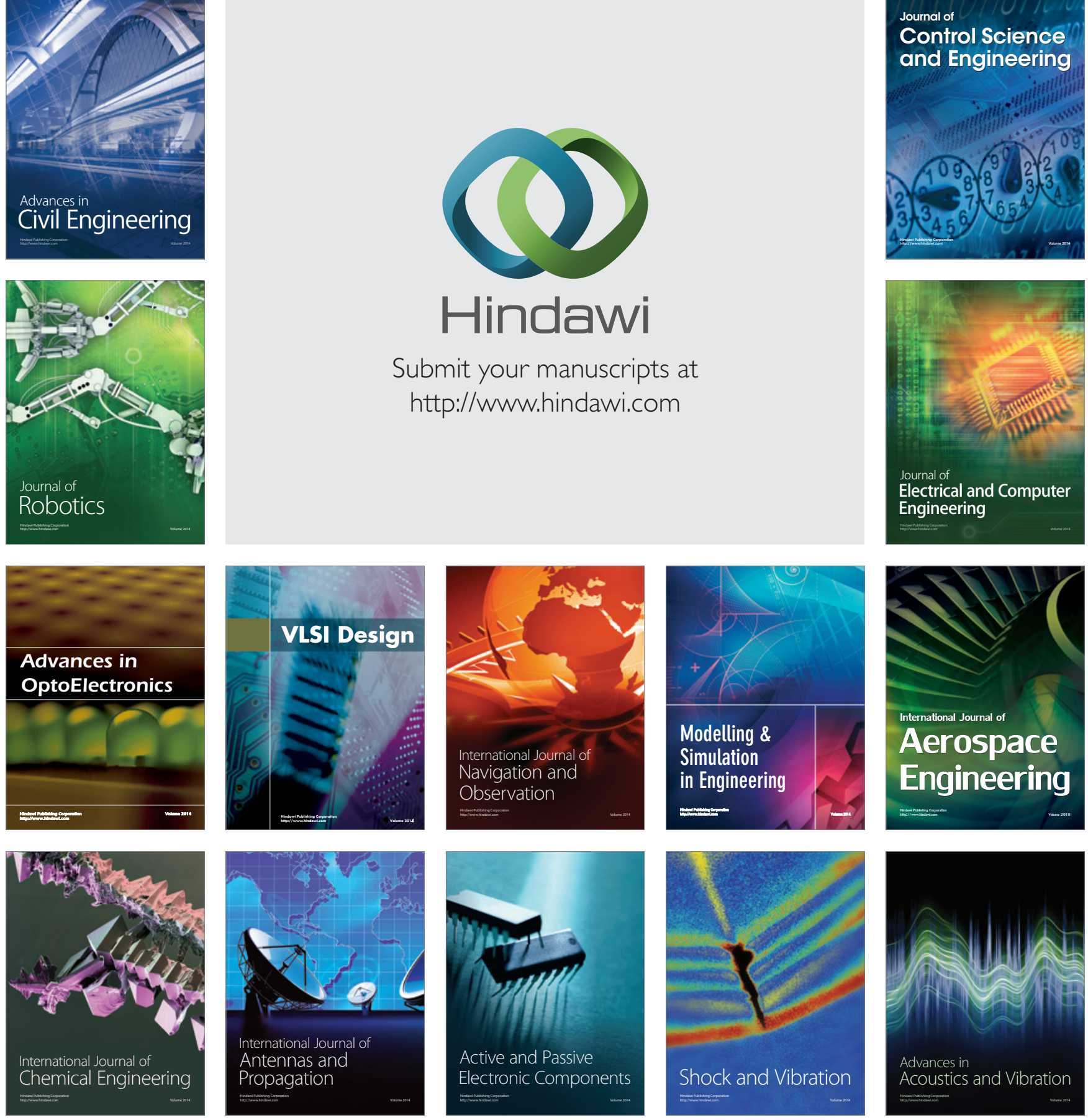